Article

\title{
Design of a New Bilayer Multipole Electromagnetic Brake System for a Haptic Interface
}

\author{
Hashim Iqbal ${ }^{1}$ and Byung-Ju Yi ${ }^{2, *}$ \\ 1 Department of Mechatronics Engineering, Hanyang University, Ansan 15588, Korea; hashim@hanyang.ac.kr \\ 2 Department of Electronic Systems Engineering, Hanyang University, Ansan 15588, Korea \\ * Correspondence: bj@hanyang.ac.kr; Tel.: +82-010-2065-5218; Fax: +82-031-401-5027
}

Received: 12 November 2019; Accepted: 5 December 2019; Published: 10 December 2019

\begin{abstract}
This paper deals with the design, simulation and experimental verification of a new bilayer multipole electromagnetic brake. The design utilizes the superposition principle of magnetic flux across the inner and outer layers of axially-oriented electromagnetic poles to provide gradual braking about the single axis of rotation. The braking principle exploits the Coulomb friction between the two rigid contact surfaces. Compared with conventional, multi-pole, multi-layer type radial brakes in haptic applications, the proposed design provides high fidelity of free motion through an absolutely disconnected rotor. The design also provides a wide operating range by delaying the saturation limit of a magnetic circuit for a wide range of input power. In this paper, the analytical model of the brake is derived and compared with the FEM-based simulation results. The optimal design obtained from multi-objective optimization was experimentally verified for its capability in haptic applications.
\end{abstract}

Keywords: multipole multilayer brakes; braking torques; multi-objective optimization; haptics; coulomb friction

\section{Introduction}

Haptic devices have become an integral part of modern human-computer interfaces, where they simulate real environments by providing realistic sensations back to a distant human operator. Recently, many useful haptic devices have spanned various applications, not limited to the automation [1], robotics [2] and medical [3] fields. Transparency and stability are the main requirements in the design of a haptic device that guarantee its high-fidelity feedback display to correctly simulate the physical environment [4]. Haptic interfaces mainly consist of active [5] and/or passive [6] actuators; those have the tendency to provide counter sensations as a result of human interaction. The key considerations in designing these actuators for haptic applications are the output forces/torques/vibrations, response time, power consumption, volume, etc. The active actuators, like electric motors though, have a fast response but they can only display a limited range of virtual impedance due to their relatively low torque to volume ratios. Their active nature also makes them vulnerable to the instabilities when interacting with the discretized components of the haptic rendering loop. Passive actuators, because of their energy-dissipating-only characteristics, are inherently stable. Their comparatively higher torque to volume ratios can display wide ranges of virtual impedance from the environment.

Electromagnetism-based passive brakes are popular choices in many haptic applications. Various designs of these brakes have been proposed in the literature that exploit the generation of eddy currents proportional to rotational velocity $[7,8]$, or the change in shear viscosity of either electrorheological (ER) fluids [9] or magnetorheological (MR) fluids [10] as a function of electric or magnetic potential, respectively. The eddy current-based brakes work for high input powers and are not suitable for low velocity applications. The rheological fluid-based brakes can provide high braking torques proportional to the sheer stress values of corresponding working fluids. In order to 
maximize the controllable braking torques in a given volume, some special designs have been proposed by researches. Those include cylindrical-shaped radial-arrangements of multiple electromagnetic poles in the form of layers [11,12]. The superposition principle of magnetic flux across the poles and layers magnifies the range of braking torque. However, these brakes tend to achieve material saturation easily that limits their operation to low ranges of input power. As the rotor is submerged in the working fluid, the operator constantly feels viscous forces at zero-input; thus, distorting the transparency of the haptic display. The unavoidable wear losses due to shear contact between fluid particles and the seal surface lead to a potential risk of fluid leakage, restricting their use in the direct human-contact applications, such as invasive surgery, rehabilitation devices, etc. [13]. Such devices require one to follow the additional safety measures mentioned as Means of Operator Protection (MOOP) in IEC-6061 standards [14].

The Coulomb friction-based electromagnetic brakes, on the other hand, utilize the variable reluctance principle to generate attraction forces on the spring-connected rotor and push it against a friction surface to apply braking. The initial isolation of rotor and stator allows free motion at zero-input. A commercially available disk-shaped design of the electromagnetic brake provides friction torque for single degree of freedom (DOF) rotation [15]. A compact EM actuator with a dual piston presses a rotor section against a conformable stator shell to provide friction braking [16]. A spherical joint-like EM actuator implements Coulomb friction-based braking in a 3-dof haptic device [17]. In these brakes, the design allows limited controllability of available braking, and the single thick solenoid coil can potentially increase the inductive time constant.

In this paper, a new bilayer multipole (BLMP) electromagnetic brake is proposed with an improved transparency alternative for haptic application. The BLMP brake utilizes the rigid contact Coulomb friction to incorporate gradual braking about single axis of rotation. The main contribution of the design is providing a zero-input transparency for haptic interface. The design also provides a wide operating range due to avoiding an early saturation of magnetic material in the presence of tiny air gaps. The input power through distributed electromagnets improves the response time due to a small inductive time constant. The capability of displaying damping and the friction of a virtual environment make it a useful device in haptics applications.

In Section 2, the mathematical models of magnetic force, braking torque, power consumption and the inductive time constant of the BLMP brake have been derived. In Section 3, a multi-objective optimization problem is defined. In Section 4, FEM based magnetostatics simulation results are presented. In Section 5, the experimental design and evaluation is presented. In Section Section 6, the results are discussed, and that is followed by the conclusion in Section 7.

\section{Modelling of a Magnetic Brake}

\subsection{Magnetic Force}

The brake contains a stator in which twelve electromagnetic poles are axially arranged in two layers. A solenoid coil is wound around each pole having a polarity opposite to its immediate neighboring poles. Six friction pads made from high friction coefficient metals are placed in spaces between these poles. A disc-shaped armature of magnetic material is placed coaxial to the stator with a tiny air gap between the two bodies, as shown in Figure 1. Because of the symmetry about the axis normal to the surface of the stator, the design is divided into six sections. Every symmetry section contains four poles with half of the actual cross-sectional area: two from inner layer and two from outer layer, as shown in Figure 2a. A schematic of a magnetic circuit is shown in Figure 3, which consists of closed loops connecting four solenoid coils through reluctances $\mathcal{R}$ and flux $\Phi$ of the magnetic path. 


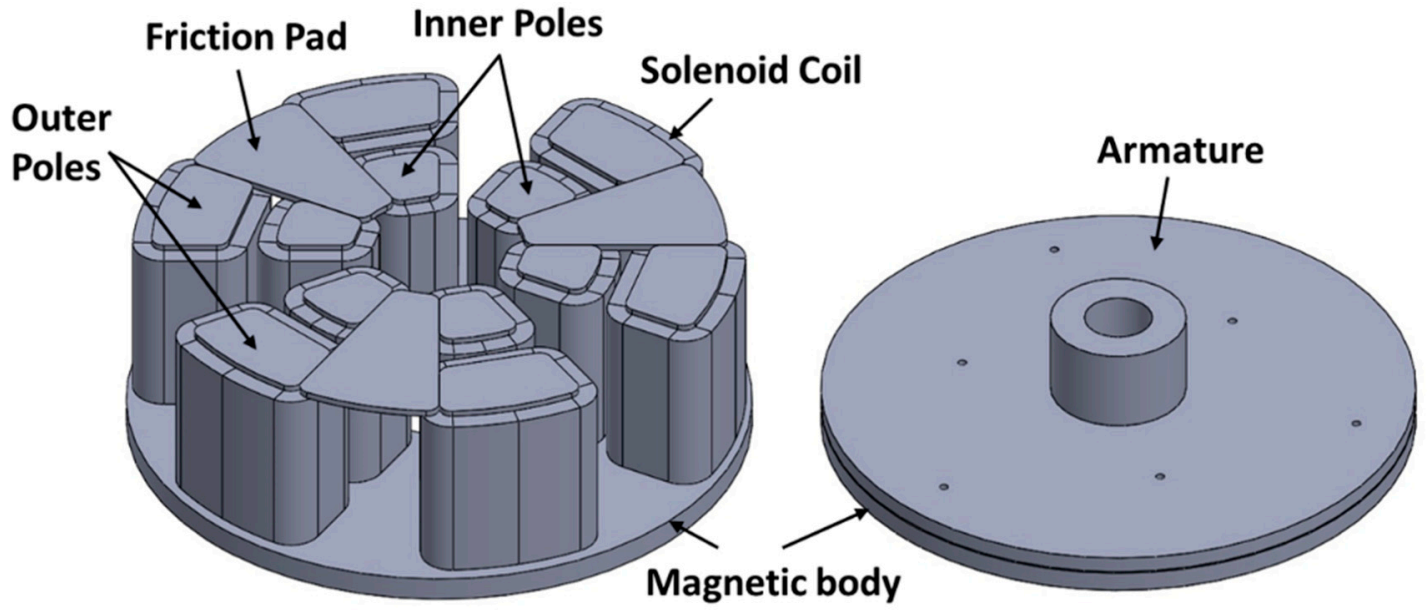

(a)

(b)

Figure 1. Design of two-layer, 12-pole axially-actuated electromagnetic brake: (a) magnetic core poles surrounded by solenoid coils and the friction pad supported by poles; (b) rotary armature with a spring-connected disc.

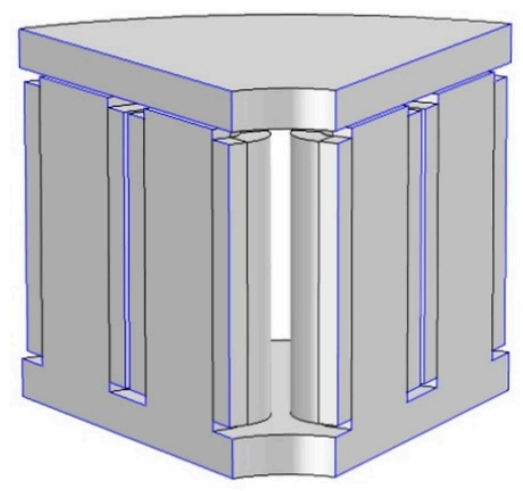

(a)

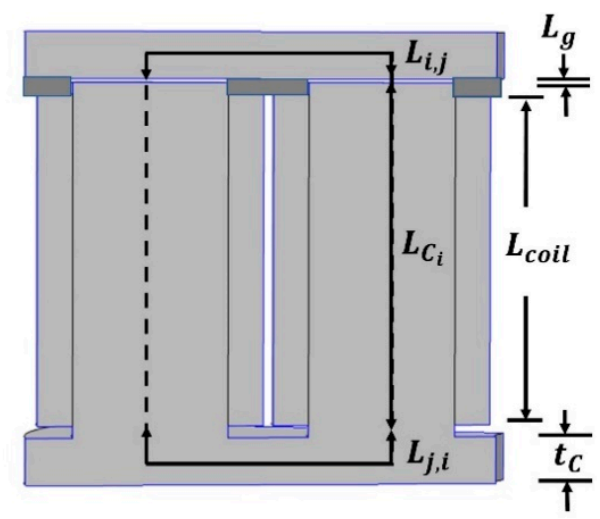

(b)

Figure 2. Layout of a symmetric section: (a) isometric view showing two inner and two outer poles; (b) side view showing a closed-path of magnetic flux.

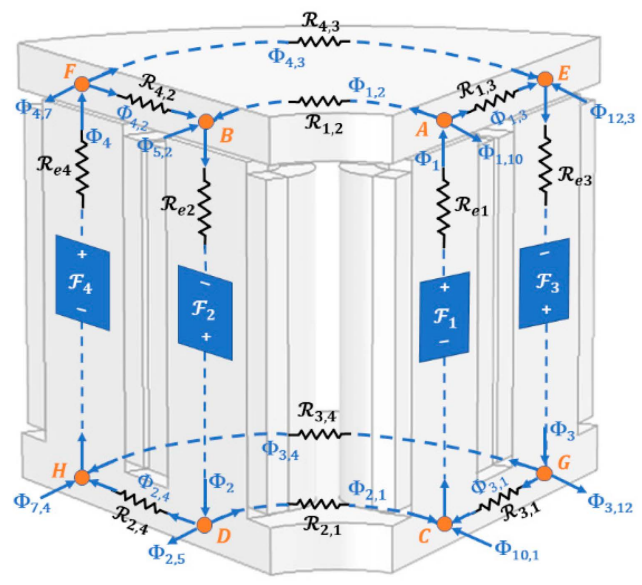

Figure 3. Schematic diagram of flux path interaction for one symmetry section. 
With $N$ turns of the solenoid coil and $I$ ampere current through the windings, the magneto-motive force $\mathcal{F}$ for each pole is given by

$$
\mathcal{F}=N I=\mathcal{R} \Phi .
$$

Analyzing the Ampere's circuit law in each closed loop of Figure 3, the superpositions of magnetic flux generated by the magneto-motive force in loops ABDCA, AEGCA, BDHFB and FEGHF, respectively, are given by the relations.

$$
\begin{aligned}
& \mathcal{F}_{1}+\mathcal{F}_{2}=\mathcal{R}_{e 1} \Phi_{1}+\mathcal{R}_{e 2} \Phi_{2}+\mathcal{R}_{1,2} \Phi_{1,2}+\mathcal{R}_{2,1} \Phi_{2,1} \\
& \mathcal{F}_{1}+\mathcal{F}_{3}=\mathcal{R}_{e 1} \Phi_{1}+\mathcal{R}_{e 3} \Phi_{3}+\mathcal{R}_{1,3} \Phi_{1,3}+\mathcal{R}_{3,1} \Phi_{3,1} \\
& \mathcal{F}_{2}+\mathcal{F}_{4}=\mathcal{R}_{e 2} \Phi_{2}+\mathcal{R}_{e 4} \Phi_{4}+\mathcal{R}_{2,4} \Phi_{2,4}+\mathcal{R}_{4,2} \Phi_{4,2} \\
& \mathcal{F}_{3}+\mathcal{F}_{4}=\mathcal{R}_{e 3} \Phi_{3}+\mathcal{R}_{e 4} \Phi_{4}+\mathcal{R}_{3,4} \Phi_{3,4}+\mathcal{R}_{4,3} \Phi_{4,3}
\end{aligned}
$$

where $\mathcal{R}_{e i}$ is the equivalent reluctance of magnetic core and air gap in the $i$ th pole. $\mathcal{R}_{i, j}$ and $\mathcal{R}_{j, i}$ are the reluctances of armature and the base, respectively, and can be calculated using Figure $2 \mathrm{~b}$ as

$$
\mathcal{R}_{e i}=\frac{L_{C_{i}}}{\mu_{0} \mu_{r} A_{C_{i}}}+\frac{L_{g_{i}}}{\mu_{0} A_{g_{i}}}, \mathcal{R}_{i, j}=\frac{L_{i, j}}{\mu_{0} \mu_{r} A_{i, j}}, \mathcal{R}_{j, i}=\frac{L_{j, i}}{\mu_{0} \mu_{r} A_{j, i}},
$$

where $\mu_{0}$ and $\mu_{r}$ are the permeability of air and relative permeability of magnetic material, respectively. The parameters $A_{C_{i}}, A_{g_{i}}, A_{i, j}$ and $A_{j, i}$ represent the cross-sectional areas of the pole, air gap, armature and base part of the magnetic circuit, respectively and their values can be calculated from the dimensional parameters illustrated in Figures $2 \mathrm{~b}$ and 4 as.

$$
\begin{gathered}
A_{C_{1}}=A_{C_{2}}=2(\text { Sector oab }-\Delta \text { ocd })=2\left(\frac{1}{2} r_{i_{\max }}^{2} \theta_{C}-\frac{1}{2} r_{i_{\min }}^{2} \tan \theta_{C}\right) \\
A_{C_{3}}=A_{C_{4}}=2(\text { Sector oef }-\Delta \text { ogh })=2\left(\frac{1}{2} r_{o_{\max }}^{2} \theta_{C}-\frac{1}{2} r_{o_{\min }}^{2} \tan \theta_{C}\right) \\
A_{1,2}=A_{2,1}=\left(r_{i_{\max }}-r_{i_{\min }}\right) t_{C} \\
A_{3,4}=A_{4,3}=\left(r_{o_{\max }}-r_{o_{\min }}\right) t_{C} \\
A_{1,3}=A_{3,1}=A_{2,4}=A_{4,2}=\frac{\theta_{S}}{2} r_{a v} t_{C} \\
A_{g_{i}}=A_{C_{i}} f_{i}
\end{gathered}
$$

where $r_{i_{\min }}, r_{i_{\max }}, r_{o_{\min }}$ and $r_{o_{\max }}$ are the minimum and maximum radial dimensions of inner and outer poles, respectively. $\theta_{C}$ and $\theta_{S}$ are the angular dimensions of the pole cross-section and the symmetry sector, respectively. The parameter $t_{c}$ defines the thickness of the armature and the bottom of the core body as shown in Figure $2 b$. The average radial distance between inner and outer poles is given by $r_{a v}=\left(r_{i_{\min }}+r_{i_{\max }}+r_{o_{\min }}+r_{o_{\max }}\right) / 4$. The factor $f_{i}=1+\left(L_{g_{i}} \ln \left(2 L_{C_{i}} / L_{g_{i}}\right) / \sqrt{A_{C_{i}}}\right)$ considers the losses due to fringing effect of magnetic flux in the air gap and depends on the geometry of the magnetic pole [18].

The parameters $L_{C_{i}}, L_{g_{i}}, L_{i, j}$ and $L_{j, i}$ are the effective lengths of the pole, air gap, armature and base part of the magnetic circuit, respectively, as calculated from Figures $2 b$ and 4 as

$$
\begin{gathered}
L_{1,2}=L_{2,1}=\theta_{S}\left(\frac{r_{\min }+r_{i_{\max }}}{2}\right) \\
L_{3,4}=L_{4,3}=\theta_{S}\left(\frac{r_{o_{\min }}+r_{o_{\max }}}{2}\right) \\
L_{1,3}=L_{3,1}=L_{2,4}=L_{4,2}=\left(\frac{r_{o_{\min }}+r_{o_{\max }}}{2}\right)-\left(\frac{r_{i_{\min }}+r_{i_{\max }}}{2}\right) .
\end{gathered}
$$

The nodal analysis at node A of magnetic circuit in Figure 3 yields that

$$
\Phi_{1}=\Phi_{1,2}+\Phi_{1,3}+\Phi_{1,10}
$$


Because of the structural symmetry, 1,10 is same as 1,2 , and therefore, Equation (6) can be re-written into

$$
\Phi_{1}=2 \Phi_{1,2}+\Phi_{1,3}
$$

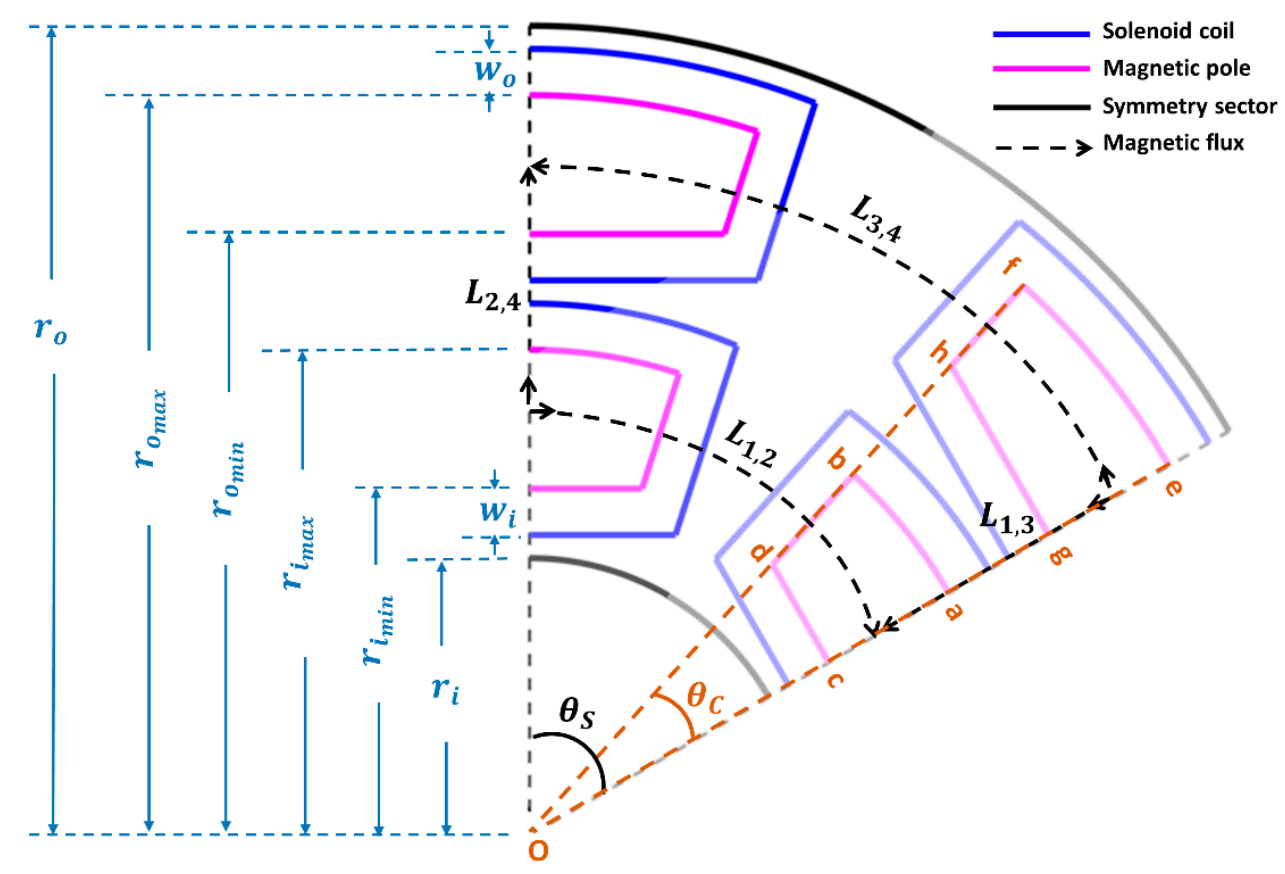

Figure 4. Nomenclature of radial dimensions for one symmetry section.

Similarly, the nodal analysis of magnetic flux at nodes B, C, D, E, F, G and H, respectively, yields

$$
\begin{gathered}
\Phi_{2}=2 \Phi_{1,2}+\Phi_{4,2}, \Phi_{1}=2 \Phi_{2,1}+\Phi_{3,1}, \Phi_{2}=2 \Phi_{2,1}+\Phi_{2,4} \\
\Phi_{3}=2 \Phi_{4,3}+\Phi_{1,3}, \Phi_{4}=2 \Phi_{4,3}+\Phi_{4,2} \\
\Phi_{3}=2 \Phi_{3,4}+\Phi_{3,1}, \Phi_{4}=2 \Phi_{3,4}+\Phi_{2,4} .
\end{gathered}
$$

The linear Equations (2), (7) and (8) can be solved for the unknown magnetic flux in each part of the magnetic circuit.

The magnetic energy stored in the volume $V_{g_{i}}=L_{g_{i}} A_{g_{i}}$ of the air-gap between the poles and the armature is given by the relationship

$$
W_{\text {mag }}=\frac{1}{2} \sum_{i=1}^{4} B_{g_{i}} H_{g_{i}} V_{g_{i}},
$$

where $B_{g_{i}}$ and $H_{g_{i}}$ are the magnetic flux density and magnetic field strength in the air gap of $i^{\text {th }}$ pole, respectively, and are related by the relation $H_{g_{i}}=B_{g_{i}} / \mu_{0}$. The magnetic flux continuity relationship $\Phi_{i}=B_{C_{i}} A_{C_{i}}=B_{g_{i}} A_{g_{i}}$ tells that the flux passing through the core and air gap is the same, hence, (9) can be re-written in the form as

$$
W_{\text {mag }}=\frac{L_{g_{i}}}{2 \mu_{0}} \sum_{i=1}^{4}\left(\frac{\Phi_{i}^{2}}{A_{g_{i}}}\right) .
$$

The symmetry section of magnetic brake allows only a half of the magnetic flux $\Phi_{i} / 2$ to pass through a half of the cross-sectional area $A_{g_{i}} / 2$ of each air gap. This magnetic flux observes a variation in 
reluctance along the path due to different permeabilities of magnetic material and air; hence, generating a pulling force $F_{m a g}=-\partial W_{m a g} / \partial L_{g_{i}}$ on the armature as a function of air gap length, formulated as

$$
F_{m a g}=-\frac{6}{4 \mu_{0}} \sum_{i=1}^{4}\left(\frac{\Phi_{i}^{2}}{A_{g_{i}}}\right) .
$$

The relation in Equation (11) considers the total magnetic force exerted by all six symmetrical parts of the magnetic brake and linearly depends on the square of magnetic flux generated in each pole.

\subsection{Braking Torque}

When the magnetic brake is actuated, the magnetic force $F_{m a g}$ pulls the rotating disc against a friction pad to seize the motion of armature. The disc is attached with the armature through a spring of known stiffness. In the off-state, the spring restoring force $T_{s p}$ keeps the friction contact dis-engaged and allows the armature to move freely.

During engaging, the normal force $F_{N}=F_{m a g}-T_{s p}$, acting at a radius $r$ on an infinitesimal contact area, generates a braking torque as given by

$$
d T_{B r}=r \mu_{K} p d A,
$$

where $\mu_{K}$ is the co-efficient of kinetic friction between the contact surfaces and the parameter $p$ is the pressure on the contact area. For a hollow circular disc having $r_{i}$ and $r_{o}$ as inner and outer radii, respectively, the net braking torque can be calculated by integrating Equation (12) for the whole contact area, as given by

$$
T_{B r}=\int_{r_{i}}^{r_{0}} 2 \pi r^{2} \mu_{K} p d r .
$$

As the pressure is uniform for the whole area of the flat contact surface, the relationship in Equation (13) can be simplified to obtain an analytical form:

$$
T_{B r}=\frac{2}{3} \mu_{K}\left(F_{m a g}-T_{S p}\right)\left(\frac{r_{o}^{2}+r_{o} r_{i}+r_{i}^{2}}{r_{i}+r_{o}}\right) .
$$

The relation in Equation (14) confers that the braking torque depends on the radial dimensions of the friction surface without being influenced by angular dimensions. But a larger surface area has an advantage of better heat dissipation [6].

\subsection{Power Consumption}

The designed multi-layered magnetic brake is operated using DC input power which is supplied to the individual solenoid coil in each layer. The solenoid coils are arranged in such a way that each pole finds its neighboring poles with inverted coil orientations. As a result, the current passing through one coil generates a flux that superimposes the flux in other poles. Since each layer of the brake can be individually actuated, all the coils in one layer have the same construction, number of turns and current.

The total power consumption by the brake is given by

$$
P_{\text {cons }}=6\left(I_{i}^{2} R_{i}+I_{o}^{2} R_{o}\right)
$$


where $I_{i}$ and $I_{0}$ are the DC currents through the solenoid coils of inner and outer layer, respectively. $R_{i}$ and $R_{o}$ are the resistances of the coil wire in the corresponding layers and can be calculated by the relation

$$
\begin{aligned}
R_{i} & =\rho \frac{\left(L_{\text {mean }}\right)_{i} N_{i}}{A_{\text {wire }}} \\
R_{o} & =\rho \frac{\left(L_{\text {mean }}\right)_{o} N_{o}}{A_{\text {wire }}},
\end{aligned}
$$

where $\rho$ and $A_{\text {wire }}$ are the resistivity and cross-sectional area of solenoid coil wire, respectively. $\left(L_{m e a n}\right)_{i}$ and $\left(L_{\text {mean }}\right)_{o}$ are the mean lengths of wire of solenoid coils in inner and outer layer, respectively, and can be calculated from the relation

$$
L_{\text {mean }}=2(U+D+b),
$$

where $U, D$ and $b$ are the lengths along the perimeter of the center of solenoid coil, as shown in Figure $5 \mathrm{a}$, with the values calculated geometrically for the inner layer solenoid as

$$
\begin{gathered}
U_{i}=\left(r_{i_{\max }}+\frac{w_{i}}{2}\right) \theta_{w_{i}} \\
D_{i}=\frac{1}{\cos \theta_{C}}\left(\left(r_{i_{\max }}+\frac{w_{i}}{2}\right) \cos \theta_{w_{i}}-\left(r_{i_{\min }}-\frac{w_{i}}{2}\right)\right) \\
b_{i}=r_{i_{\min }} \tan \theta_{C}+\frac{w_{i}}{2}\left(\frac{1}{\cos \theta_{C}}-\tan \theta_{C}\right) \\
\theta_{w_{i}}=\theta_{C}+\sin ^{-1}\left(\frac{w_{i}}{2 r_{i_{\max }}+w_{i}}\right),
\end{gathered}
$$

where $w_{i}$ is the width of solenoid coil in the inner layer. Similarly, the mean length for outer layer solenoid can also be calculated using Equation (18).

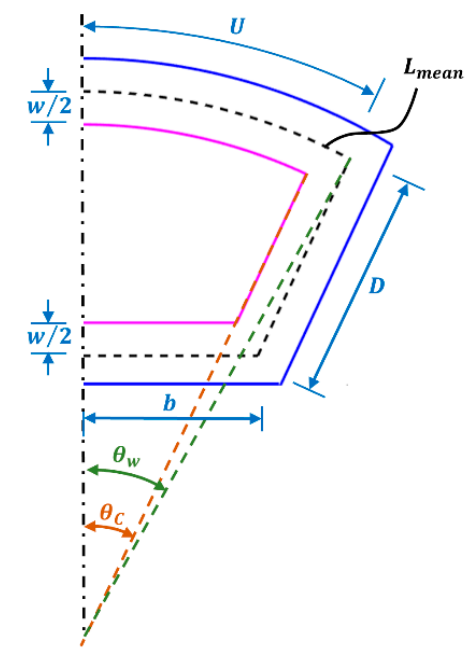

(a)

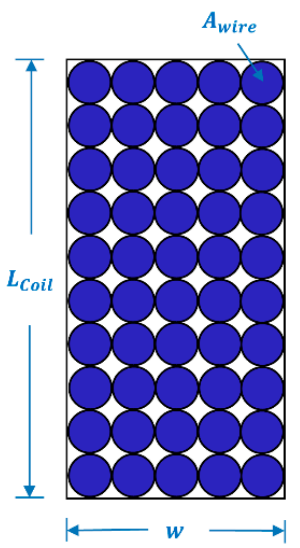

(b)

Figure 5. Schematic diagram for a single solenoid coil. (a) Radial dimensions; (b) cross-sectional dimensions.

The number of turns of solenoid coil can be calculated by estimating the number of circles of cross-sectional area $A_{\text {wire }}$ that can fit in an available area-width $w$ and length $L_{\text {coil }}$ of a solenoid coil, as shown in Figure 5b, and expressed by the relation

$$
N=\frac{w L_{\text {coil }} \delta}{A_{\text {wire }}}
$$

where $\delta=\pi / \sqrt{12}$ is the coil packing factor that considers the free spaces between the wrapped wires [19]. 


\subsection{Inductive Time Constant}

The electric circuits which are comprised of inductors do not pass current instantaneously. The term inductive time constant is defined as the time required by the current passing through the solenoid circuit to reach $63 \%$ of its maximum value. The current will eventually reach its maximum value after a period of five-time constants, and the same is applicable when the current is switched OFF. For the application where faster response is required for the system, the lower value should be its inductive time constant. The inductive time constant TC of solenoid circuits in the inner and outer layers of magnetic brake is given by the relation

$$
T C_{i}=\frac{\mathrm{L}_{i}}{R_{i}} T C_{o}=\frac{\mathrm{L}_{o}}{R_{o}}
$$

where $\mathrm{L}_{i}$ and $\mathrm{L}_{o}$ are the inductances of solenoid coils in the inner and outer layers, respectively. The value of these inductances depends on the amount of current through the wire, magnetic flux through pole and the number of turns of the coil, as given by the expressions:

$$
\mathrm{L}_{i}=\frac{N_{i} \Phi_{j}}{I_{i}}, \text { where } \Phi_{j}=1,2 \mathrm{~L}_{o}=\frac{N_{o} \Phi_{k}}{I_{o}}, \text { where } \Phi_{k}=3,4
$$

It has been noted from Equation (20) that an increase in coil resistance can further improve the response of the system but at the cost of additional power dissipation formulated in Equation (15).

\section{Multi-Objective Optimization}

An effective electromagnetic brake can provide large magnetic force that can generate large braking torque at the expense of small input power. It is also desirable to have faster response, particularly for the applications where time-delays adversely affect the efficiency of a task. The analytical models developed in Section II realize the fact that, for a constrained volume of the brake, the more which is the coil current, the more it generates magnetic force, but also increases the power consumption. Alternatively, a greater number of turns of the coil can limit the input power, but it also increases the inductive TC; hence, brake response is degraded.

In order to come up with an optimum design that can best deal with these conflicting properties, a multi-objective optimization procedure is proposed. For a fixed volume of electromagnetic brake, the objective is to maximize the magnetic force $F_{m a g}$ while minimizing the inductive TC and the power consumption $P_{\text {cons }}$. The optimization procedure is formulated as

$$
\begin{gathered}
x=\left[\theta_{C}, R_{i_{\min }}, R_{i_{\max }}, R_{o_{\min }}, R_{o_{\max }}, w_{i}, w_{o}\right]^{T}, \text { with } x_{\min } \leq x \leq x_{\max } \\
\text { Maximize }: f(x)=\alpha \frac{F_{\operatorname{mag}}}{F_{\text {norm }}}+\beta \frac{T C_{\text {norm }}}{\max \left(T C_{i}, T C_{o}\right)}+\gamma \frac{P_{\text {norm }},}{P_{\text {cons }},}, \\
\text { Subject to }: r_{i}<R_{i_{\min }}<R_{i_{\max }}<R_{o_{\min }}<R_{o_{\max }}<r_{o}, \\
\Phi_{i} \leq B_{\text {sat }} A_{g_{i}},
\end{gathered}
$$

where $x$ is the vector of design variables, shown in Figure 4, which are bounded by lower and upper values, as described by $x_{\min }$ and $x_{\max }$, respectively. The objective function is comprised of the scaled and normalized sum of magnetic force, inductive time constant and power consumption. $F_{\text {norm }}, T C_{\text {norm }}$ and $P_{\text {norm }}$ are the normalized values obtained through the optimization of individual parameters in the given constraints, and they were chosen as $133 \mathrm{~N}, 20 \mathrm{~ms}$ and $1.5 \mathrm{~W}$, respectively. The scaling terms $\alpha, \beta$ and $\gamma$ are chosen according to the priority given to the brake properties, and their values were selected as $0.5,0.3$ and 0.2 respectively. The proposed design of magnetic brake has many tiny air-gaps in its magnetic circuit which allow it to operate even for greater range of input power. Because of the limitation of maximum allowable magnetic flux density $B_{s a t}$, the most important requirement is to achieve maximum possible magnetic force that can generate a large braking torque before the device 
gets saturated. The brake was being designed focusing on its application in haptics; therefore, a faster response would have much more importance.

A sequential quadratic programming (SQP) method was adopted to carry out an optimization procedure. SQP is a gradient-based optimization algorithm that leads the objective function towards the nearest minimum in the neighborhood of the chosen initial point. The optimization procedure was input with initial values of the design variables, taken from the parameters of initial design of BLMP brake, shown in Figure 6a. The procedure took five iterations to reach the final values, suggesting the optimal design shown in Figure $6 \mathrm{~b}$. For a fixed current supply to the coils of each layer, the cross-sectional area of the poles was adjusted in the optimal design to provide more space to solenoid coils and minimize the distance between the poles. A comparison of the initial and optimal design variables is shown in Table 1.

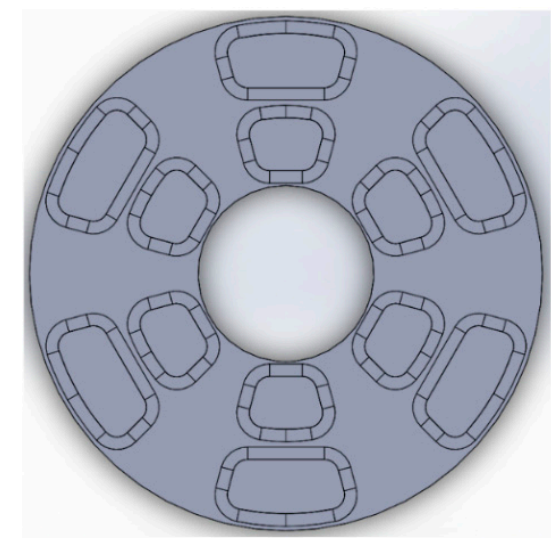

(a)

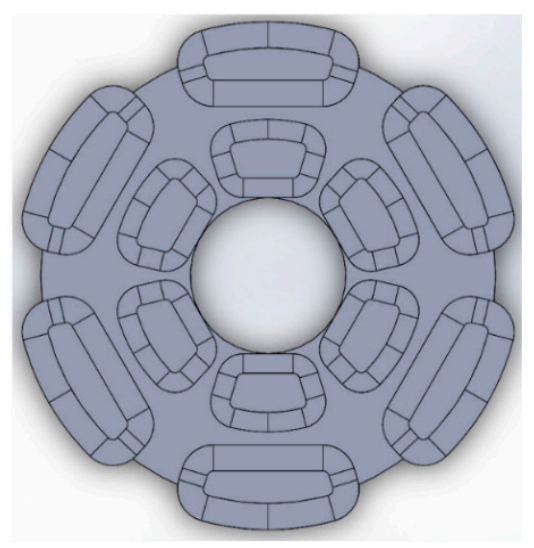

(b)

Figure 6. Design evolution during optimization procedure. (a) Initial design; (b) optimal design.

Table 1. Initial and optimal values of design variables for the new bilayer multipole (BLMP) brake.

\begin{tabular}{|c|c|c|c|c|c|c|c|c|}
\hline $\begin{array}{c}\text { Design } \\
\text { Variables }\end{array}$ & $\begin{array}{c}\theta_{C} \\
(\mathrm{deg})\end{array}$ & $\begin{array}{l}R_{i_{\min }} \\
(\mathrm{mm})\end{array}$ & $\begin{array}{l}R_{i_{\max }} \\
(\mathrm{mm})\end{array}$ & $\begin{array}{l}R_{O_{\min }} \\
(\mathrm{mm})\end{array}$ & $\begin{array}{l}R_{O_{\max }} \\
(\mathrm{mm})\end{array}$ & $\begin{array}{c}w_{i} \\
(\mathrm{~mm})\end{array}$ & $\begin{array}{c}w_{o} \\
(\mathrm{~mm})\end{array}$ & $f(x)$ \\
\hline Initial values & 15 & 14 & 21.2 & 25.3 & 32.7 & 1.7 & 1.7 & 0.3 \\
\hline Optimal values & 17 & 15 & 21 & 30 & 35 & 2.5 & 3.5 & 0.69 \\
\hline
\end{tabular}

\section{FEM-Based Magnetostatics Simulations}

The performance of the optimal design of BLMP brake has been validated using finite element method (FEM) based simulation software, named COMSOL Multiphysics. The magnetostatics simulation was carried in a 3-dimensional brake model. A magnetic material with a known magnetization curve was selected for the core domain surrounded by the air with relative permeability equal to 1 . The same value of DC current was supplied to all the multi-turn solenoid coil domains of individual layer that result in the generation of magnetic flux moving along the magnetic circuit of Figure 3. Upon controlling the actuation of each layer individually, there is a superposition of magnetic flux in the armature domain that connects the flux of each pole with surrounding poles. The direction of magnetic flux has been shown in Figure 7, when applying a current of $1 \mathrm{~A}$ to each coil of the layer. Due to the actuation of inner layer only, the magnetic flux flows through the inner poles of the brake due to the shortest distance in between them, as shown in Figure 7a. Because of having a greater number of turns and thicker cross-section poles at the outer layer, the magnetic flux connects both the inner and outer layer poles when actuating the outer layer only, and further strengthens the same path when the combination of two layers is actuated, as shown in Figure $7 \mathrm{~b}, \mathrm{c}$, respectively. The simulation results validate the proposed arrangement of solenoid coils in the brake design and the sequence of actuating individual layer to maximize the distribution of magnetic flux in the material. 
The thick cross-sectional poles help in generating larger magnetic flux densities proportional to the input solenoid current unless the magnetic material gets saturated. The presence of large number of tiny air gaps in the magnetic circuit can delay the saturation stage for higher values of input currents; therefore, a wide range of magnetic forces is available. For an air gap of $0.1 \mathrm{~mm}$, a comparison of magnetic flux density distribution is shown in Figure 8 when applying a current of $1 \mathrm{~A}$ to each coil of the layer. The simulation results describe the uniform distribution of magnetic flux densities along the poles and the armature during all the actuation sequences, and hence, validate the optimal design of BLMP brake.

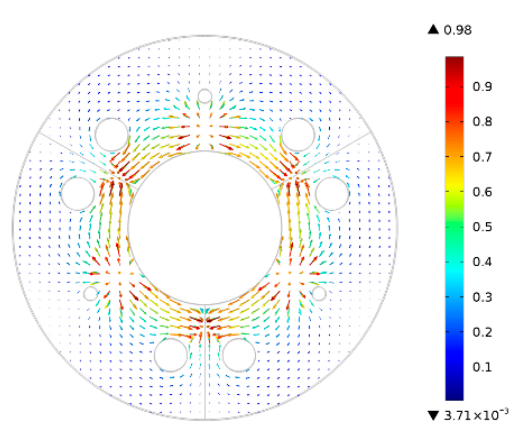

(a)

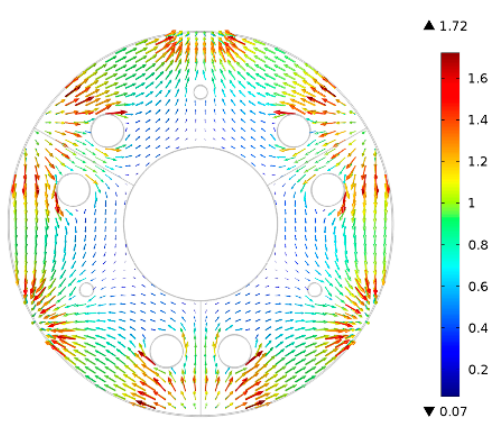

(b)

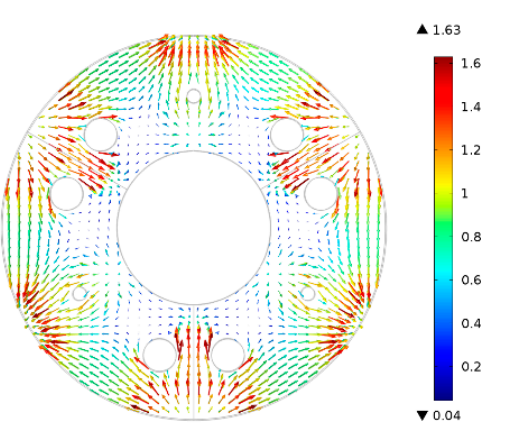

(c)

Figure 7. Flow direction of magnetic flux in the armature domain with the actuation of (a) Inner layer only; (b) Outer layer only; (c) Combination of inner and outer layer.

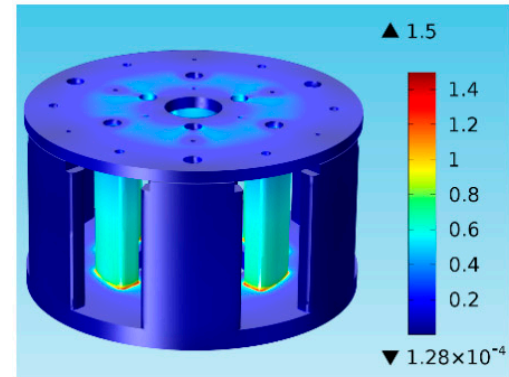

(a)

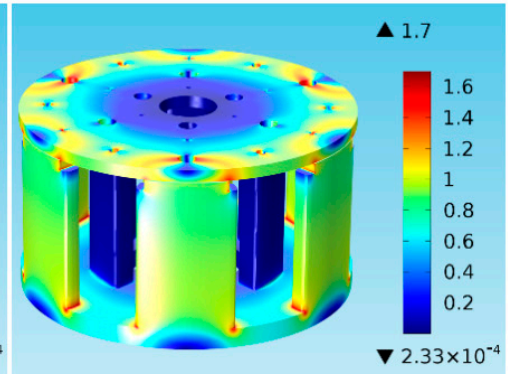

(b)

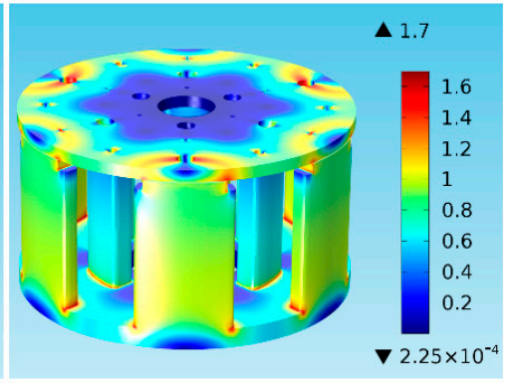

(c)

Figure 8. Magnetic flux density distribution along the cross-section of design with the actuation of the (a) inner layer only; (b) the outer layer only; (c) a combination of the inner and outer layers.

Figure 9 shows a comparison of the analytical model of a brake with simulation results, wherein the magnetic forces are plotted as a function of air gap during actuation of the inner and combined layer for an input current of $1 \mathrm{~A}$ to each coil. The result shows that a gradual pulling force can be achieved from the brake design by controlling the current in each layer, sequence of actuation of the layers and the air gap between the armature and the friction pad. 


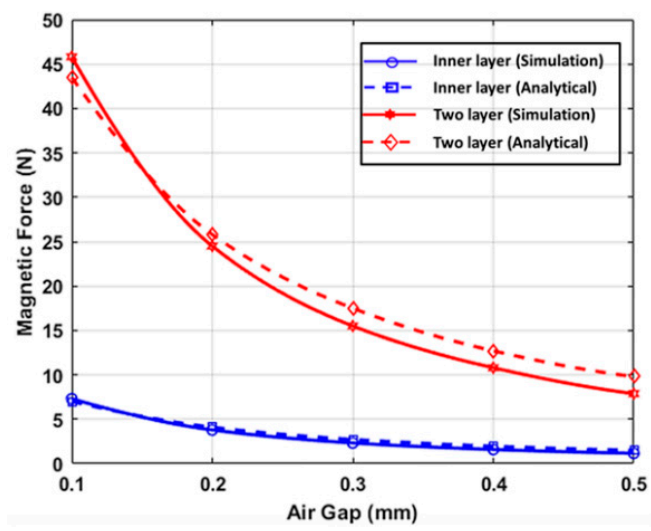

Figure 9. Magnetic force as a function of the air gap. A comparison of simulation results with analytical model, for individual and combined actuation of layers.

\section{Experimental Evaluation}

\subsection{Experimental Setup}

A prototype of the BLMP brake was manufactured for experimental verification of its characteristics and performance, as shown in Figure 10a. The AISI 420 magnetic steel was used for the core body due to its high fatigue strength and corrosion resistance properties, specifically chosen for the cyclic loading and shearing nature of the contact surfaces. The properties in a magnetic material are induced at the expense of high coercivity in its magnetization properties. The height of each pole of the core body is $30 \mathrm{~mm}$; the remaining dimensions are given in Table 1 . The friction pads having $2 \mathrm{~mm}$ thickness are made of aluminum alloy that has a coefficient of friction of about $\mu=0.45$ with the steel, and they generate little wear upon contact. The enameled copper wire with $0.65 \mathrm{~mm}$ diameter is used for winding $N=75$ turns on each coil of inner and outer layers. The higher resistance of AWG 22 wire helps in improving the inductive time constant of the coil. In the armature body, a $3 \mathrm{~mm}$ magnetic disc is connected with a non-magnetic rotor through a $0.3 \mathrm{~mm}$ thin sheet of 304 stainless steel that acts as a spring to allow relative motion in the air gap. The prototype of BLMP brake shown in Figure 10a weighs $646 \mathrm{~g}$ only.

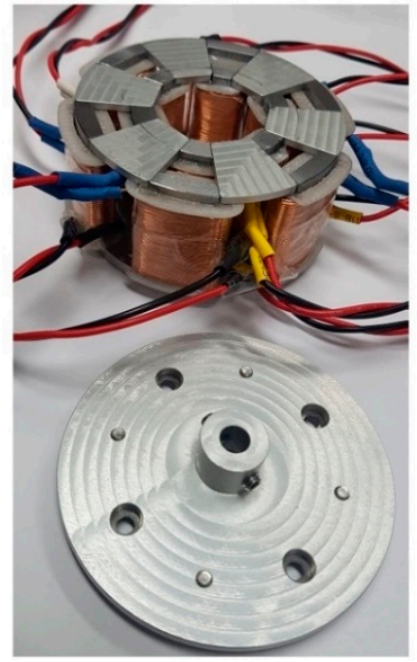

(a)

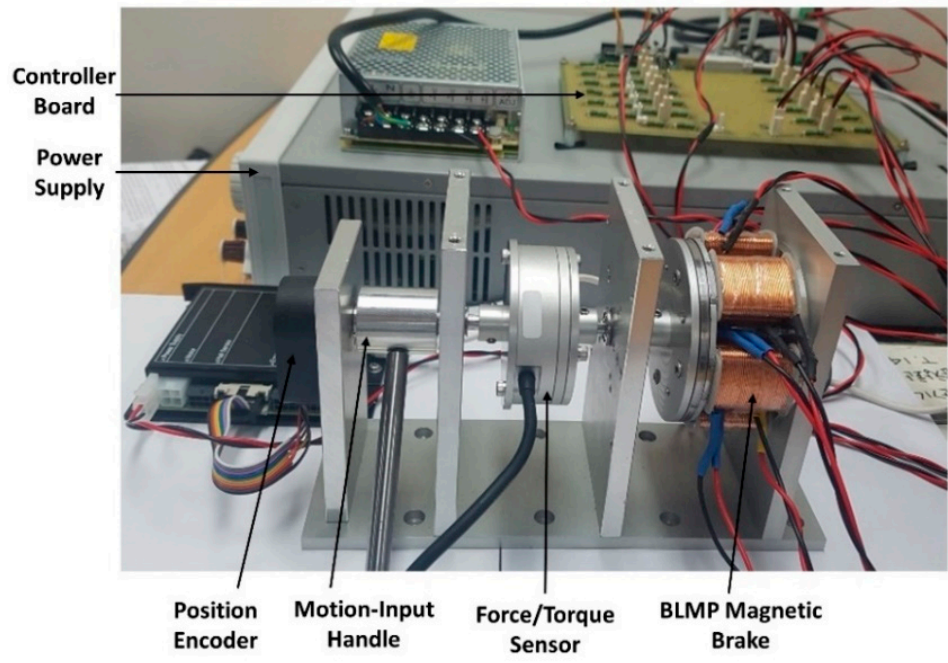

(b)

Figure 10. Experimental setup. (a) Prototype of proposed BLMP brake; (b) experimental platform for BLMP brake. 
Figure 10b shows an experimental setup using BLMP brake. The stator of the brake is fixed with a support, whereas the armature is attached with motion-input handle through a six-axis force/torque sensor by Robotous co. and a 4096-bit position encoder by US Digital co. An 80 V, 120 A DC power supply is used in current control-mode to provide input to the brake. The computer system is used for data acquisition from sensors with a sampling time of $20 \mathrm{~ms}$ and an Arduino board provides a PWM control at the rate of $31 \mathrm{kHz}$.

\subsection{Force and Torque Characteristics}

The BLMP brake was analyzed for its capability of generating a wide range of magnetic forces as a function of variable input current. For that, the experimental setup in Figure $10 \mathrm{~b}$ was used; the spring in the armature body was removed to maintain a fixed air gap of $0.5 \mathrm{~mm}$ between the poles and armature. The force sensor measured the axial direction magnetic forces acting on the armature as a result of actuating the coils of the inner layer and the combination of the two layers, shown in Figure 11a,b, respectively. Because of the unavailability of analytical hysteresis model of the material, the analytical model of magnetic force was plotted with the increasing current; only, that was validated by the experiment results.

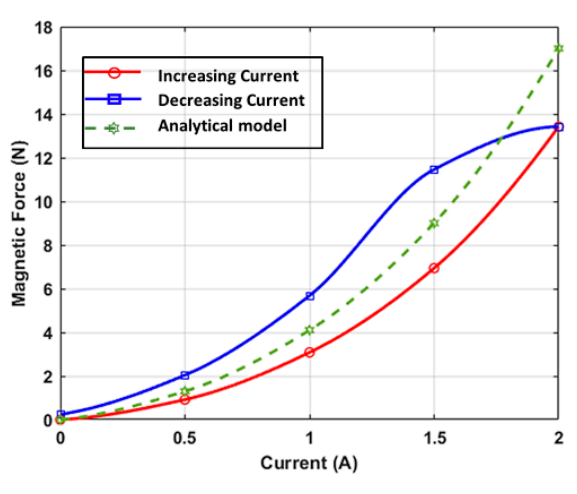

(a)

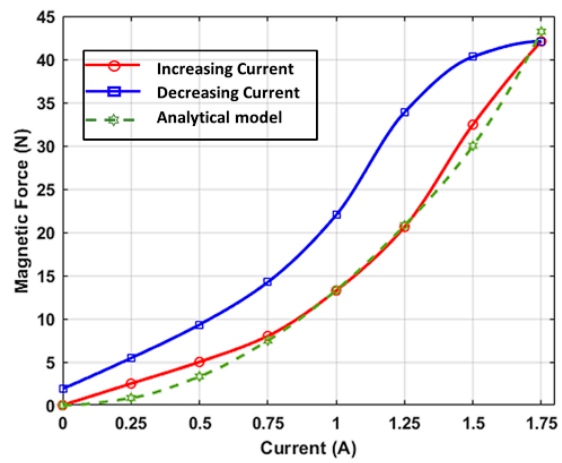

(b)

Figure 11. Magnetic pulling force on the armature as a function of coil currents with (a) inner layer actuation only; (b) combined layer actuation.

For analyzing the braking capability as a function of variable input current, the same setup of Figure $10 \mathrm{~b}$ was used with the inclusion of spring in the armature body. The armature body rotates freely, maintaining an initially fix air gap with the poles in the dis-engaged state. As the magnetic disc is attached with the armature through an unstretched-spring, an engaging force proportional to the spring restoring force $T_{s p}$ is required to pull the magnetic disc all the way through air-gap. In the dis-engaged state, there is no contact between armature and friction pad; hence, no friction torque is available. During the actuation of brake, the magnetic force pulls the spring-connected magnetic disc against the friction pads, and the engaging force becomes constant due to no further displacement of the armature, as shown in the top graphs of Figure 12. In this engaged state, the resulting braking torque that is proportional to the net normal force $F_{N}=F_{m a g}-T_{s p}$ is measured by the torque sensor when user inputs rotary motion through the handle. Figure 12 shows three cases of torque measurement, before and after engaging. Firstly, an initial air gap of $0.5 \mathrm{~mm}$ was maintained and the coils of inner layer of brake were actuated with a 0.5 A current increments, as shown in Figure 12a. Secondly, with the same initial air gap, the coils of both inner and outer layers of brake were actuated with a $0.3 \mathrm{~A}$ current increment, as shown in Figure 12b. Lastly, the initial air gap was reduced to $0.35 \mathrm{~mm}$ and a 0.3 A current increment was provided to both inner and outer layer coils of the brake, as shown in Figure 12c. The experiment results also validate the analytical model derived in Equation (14). 

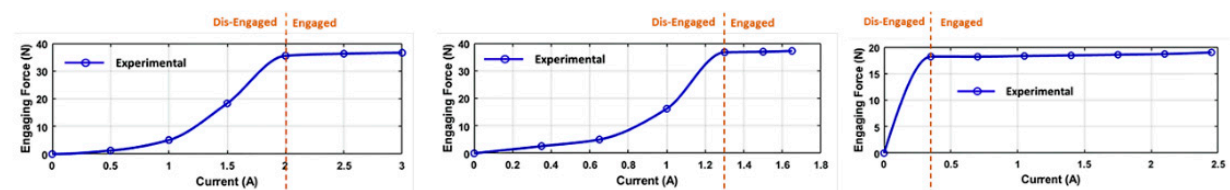

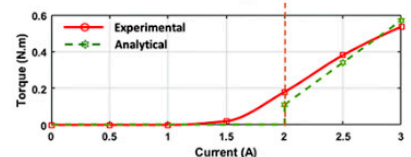

(a)

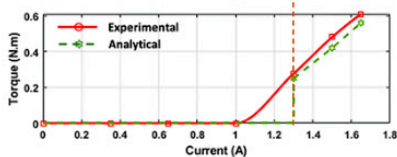

(b)

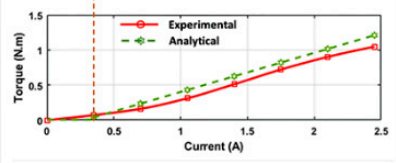

(c)

Figure 12. Brake engaging force and resulting torque as a function of coil currents with (a) inner layer actuation when the air gap was $0.5 \mathrm{~mm}$; (b) combined layer actuation when the air gap was $0.5 \mathrm{~mm}$; (c) combined layer actuation when the air gap was $0.35 \mathrm{~mm}$.

\subsection{Damping Characteristics}

The BLMP brake was analyzed for its ability to simulate virtual damping. The experimental setup was arranged similarly to the one shown in Figure 10b. A cyclic rotation motion with a frequency of $0.5 \mathrm{~Hz}$ and an amplitude of $2.1 \mathrm{rad}$ was applied to the motion-input handle. The coils of inner layer were actuated for $1 \mathrm{~A}, 1.5 \mathrm{~A}, 2 \mathrm{~A}, 2.5 \mathrm{~A}$ and $3 \mathrm{~A}$ input currents. The hysteresis loop of torque-velocity relationship in Figure 13a and the torque-position relationship in Figure 13b show the energy absorbed is proportional to current level increments, and that validates the effectiveness of the BLMP brake for uniformly simulating the virtual damping across the whole working range.

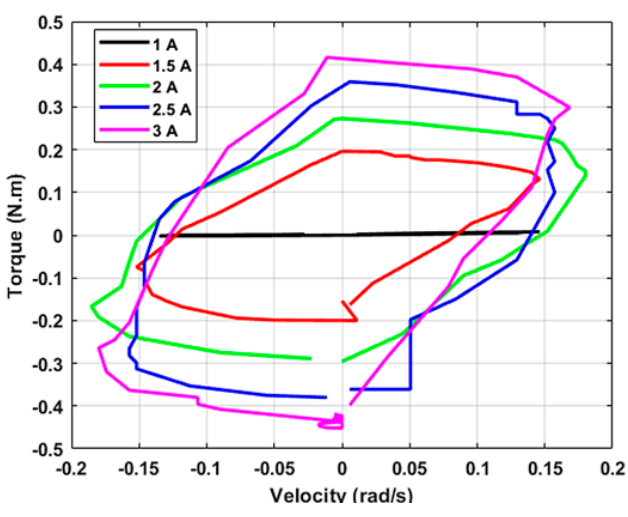

(a)

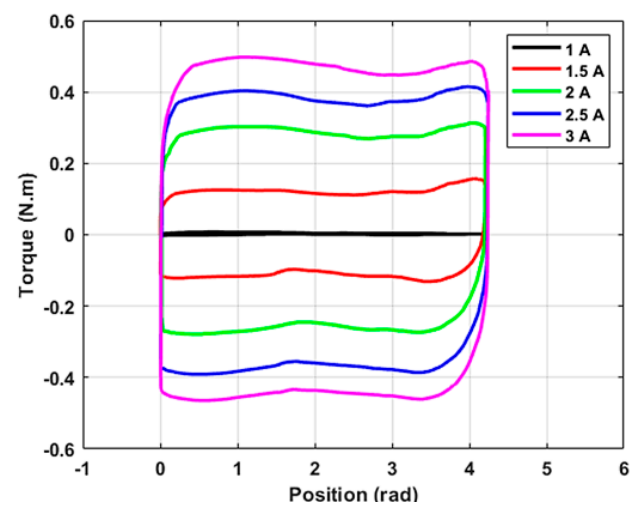

(b)

Figure 13. Damping behavior of BLMP brake at different current levels. (a) Torque-velocity characteristic curve; (b) torque-position characteristic curve.

\subsection{Transient Time Characteristics}

To investigate the transient characteristics of the BLMP brake, the experimental setup of Figure 10b was used. After being properly demagnetized, a step input of 2 A current was applied to the coils of inner layer. The results in Figure 14 exhibit the behavior of braking torque as a first-order linear system for a step input. The rise and fall time of torque curve was calculated: $0.13 \mathrm{~s}$ and $0.45 \mathrm{~s}$ respectively. The delay in fall time was due to the residual magnetization of the material that would not release the armature quickly. For that, a spike of small reverse direction current was applied at the falling step of input current. After this demagnetization technique, the fall time of torque curve was reduced to $0.3 \mathrm{~s}$. 


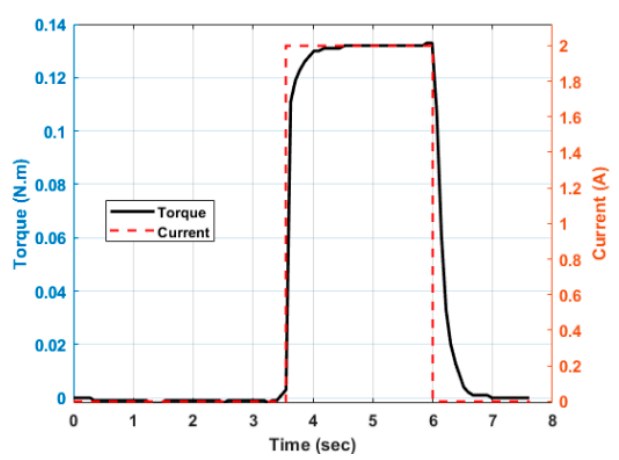

Figure 14. Transient behavior of BLMP brake for a step input current of $2 \mathrm{~A}$ to inner layer.

\subsection{Virtual Wall Collision}

An experiment was performed to assess how efficiently the BLMP brake stimulates a collision with a stiff virtual wall. The setup presented in Figure 10b was used to perform the experiment. The motion-input handle was allowed to penetrate into a virtual wall located at a position of about $2.1 \mathrm{rad}$ in several attempts. Upon reaching the wall location with different velocities, the brake was activated by a current of $2 \mathrm{~A}$ supplied to the inner layer solenoid coils. As a result, the motion energies were dissipated, and the motion-input handle was stuck at the wall position. The controller assessed a decrease in braking torque at zero velocity as an indication of the user-intention to move-away from the wall. For a decrease in braking torque below $0.05 \mathrm{Nm}$, the brake was de-activated, allowing the motion-input handle to move backward. Figure 15 shows the results of wall collision simulated by BLMP brake.

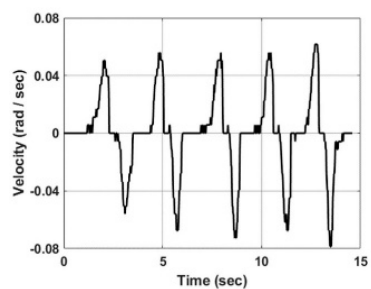

(a)

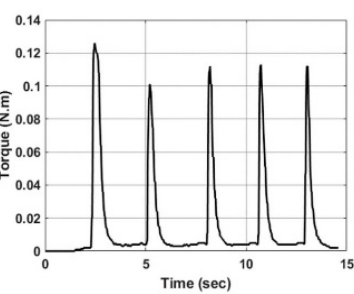

(b)

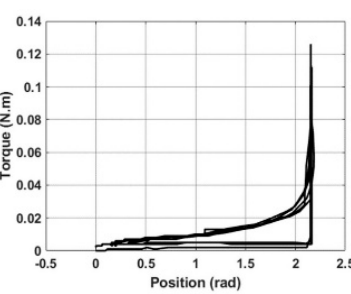

(c)

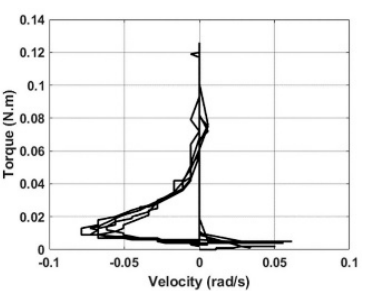

(d)

Figure 15. Simulation of a collision with a virtual wall at a position of $2.1 \mathrm{rad}$. The motion-input handle struck the wall in multiple attempts. (a) The motion-velocities along the trajectory; (b) the braking torques at the time of collisions; (c) the collision generates crisp values of Braking torques as functions of handle-position; (d) the detachment of motion-input handle occurred at zero velocity by some control input.

\section{Discussion}

Materials with high fatigue strength and corrosive resistance are suitable choices for the direct contact friction surfaces. The ferromagnetic materials having such characteristics are considered hard-magnetic materials, which show considerably large coercive fields in their B-H curves. The results in Figure 11 show a clear deviation of magnetic forces for increasing followed by decreasing values of input currents. The maximum hysteresis was measured as $4.6 \mathrm{~N}$ for the inner layer and $13.4 \mathrm{~N}$ for the combination of the two layers. The brake can be demagnetized by giving a small spike of reverse current in the off-state. Alternately, a softer magnetic material having low hysteresis can be used to provide high magnetic forces at lower inputs due to their high relative permeabilities, and also improve the response time of the brake by fast armature detachment due to its lower residual magnetism.

The results in Figure 12 show that an initial quantity of power is consumed to pull armature connected with a stiff spring. It is shown in Figure 12a that, as the current increases in the inner 
layer, the engaging force acting on the armature drastically increases with the reducing air gap. After engaging, the braking torque is then a linear function of input current. The results in Figure 12b show that, for the same initial air gap, the engaging happens at a $1.3 \mathrm{~A}$ current given to both inner and outer layer coils, whereas a steeper slope of braking torque was observed after engaging. The results in Figure 12c show that the engaging happens at a reduced current and force, resulting in larger braking torques available for the current increments onwards. The experiment also shows a small eddy current braking torque just before the actual engaging of the brake, which has a negligible effect due to lower velocity motion.

The results from the virtual wall collision experiment show large crisp values of braking torque at the wall. The brake effectively dampened all the velocities near the virtual wall, and the non-negative torques for both positive and negative velocities validate that the brake effectively escapes from the stuck situation without generating non-intuitive braking while pulling out from the wall.

The experimental results validate that the BLMP brake can render a wide range of stiffness offered by the virtual environment. The maximum value of stiffness offered is limited by the material saturation that can be delayed only in the presence of tiny air gaps in the magnetic circuits. The passive actuators can only dissipate the energy generated by the system, and without an energy restoring component, the haptic rendering cannot be fully perceived. In a future study, the BLMP brake will be interfaced with an active actuator; i.e., a motor at the joints of a multiple-degrees-of-freedom haptic device. The hybrid interface would then be capable of simulating the energy storing elements like springs.

\section{Conclusions}

This paper presents a new design of a bilayer multipole electromagnetic brake and validates its capability for haptic applications. The brake incorporates superposition of magnetic flux through the independent actuation of two layers to provide gradual braking at various levels. The design provides the transparency at zero input, which other designs cannot perfectly simulate. The design also offers a wide operating range by delaying the saturation limit of the material for large input values. The derived analytical model of the brake was used to perform multi-objective optimization. The FEM simulation was then performed to analyze, and it validated the behavior of the optimal design. The experimental results show that the magnetic properties of the material play an important part in its performance. Due to the small inductive time constant of the proposed design, a small reversed current allows a quick demagnetization of magnetic material, and this transient response can be further improved by using a material with low coercivity. It was also confirmed that a choice of a stiff spring allows a quick detachment even in the presence of residual magnetization, but, at the expense of low available braking torque. The braking of a BLMP brake was based on the principle of Coulomb friction; therefore, there was valid reasoning to say that the brake had the capability of simulating virtual friction along with virtual damping, both of which have now been verified experimentally.

Author Contributions: H.I. contributed to the conceptualization, methodology, visualization and original draft preparation; B.-J.Y. contributed to the supervision and review and editing. All the authors contributed to the paper.

Funding: This work was supported by the Technology Innovation Program (or Industrial Strategic Technology Development Program-Artificial intelligence bio-robot medical convergence project) (20001257, Artificial intelligence algorithm based vascular intervention robot system for reducing radiation exposure and achieving $0.5 \mathrm{~mm}$ accuracy) — funded by the Ministry of Trade, Industry and Energy(MOTIE, Korea), the Ministry of Health and Welfare(MOHW), the Ministry of Science and ICT (MSIT) and the Korean Evaluation Institute of Industrial Technology (KEIT); the Technology Innovation Program (10052980, Development of micro-robotic system for surgical treatment of chronic total occlusion) — funded by the Ministry of Trade, Industry and Energy (MI, Korea); and the WC300 R\&D Program (S2482672) — funded by the Small and Medium Business Administration (SMBA, KOREA).

Conflicts of Interest: The authors declare no conflict of interest. 


\section{References}

1. Kim, S.-Y.; Yang, T.-H. Miniature impact actuator for haptic interaction with mobile devices. Int. J. Control Autom. Syst. 2014, 12, 1283-1288. [CrossRef]

2. Derlukiewicz, D. Application of a Design and Construction Method Based on a Study of User Needs in the Prevention of Accidents Involving Operators of Demolition Robots. Appl. Sci. 2019, 9, 1500. [CrossRef]

3. Woo, J.; Song, H.; Cha, H.-J.; Yi, B.-J. Advantage of Steerable Catheter and Haptic Feedback for a 5-DOF Vascular Intervention Robot System. Appl. Sci. 2019, 9, 4305. [CrossRef]

4. Jinung, A.; Dong-soo, K. Haptic experimentation on a hybrid active/passive force feedback device. In Proceedings of the 2002 IEEE International Conference on Robotics and Automation, Washington, DC, USA, 10-17 May 2002; Volume 4, pp. 4217-4222.

5. Bai, K.; Ji, J.; Lee, K.-M.; Zhang, S. A two-mode six-DOF motion system based on a ball-joint-like spherical motor for haptic applications. Comput. Math. Appl. 2012, 64, 978-987. [CrossRef]

6. Goo, B. A Study on the Contact Pressure and Thermo-Elastic Behavior of a Brake Disc-Pad by Infrared Images and Finite Element Analysis. Appl. Sci. 2018, 8, 1639. [CrossRef]

7. Berkelman, P.J.; Hollis, R.L. Lorentz Magnetic Levitation for Haptic Interaction: Device Design, Performance, and Integration with Physical Simulations. I. J. Robot. Res. 2000, 19, 644-667. [CrossRef]

8. Gosline, A.; Hayward, V. Eddy Current Brakes for Haptic Interfaces: Design, Identification, and Control. IEEE/ASME Trans. Mechatron. 2008, 13, 669-677. [CrossRef]

9. Bose, H.; Berkemeier, H.-J. Haptic Device Working with an Electrorheological Fluid. J. Intell. Mater. Syst. Struct. 1999, 10, 714-717. [CrossRef]

10. Li, W.; Du, H. Design and Experimental Evaluation of a Magnetorheological Brake. Int. J. Adv. Manuf. Technol. 2003, 21, 508-515. [CrossRef]

11. Shiao, Y.; Ngoc, N.; Lai, C.-H. Optimal design of a new multipole bilayer magnetorheological brake. Smart Mater. Struct. 2016, 25, 115015. [CrossRef]

12. Wu, J.; Li, H.; Jiang, X.; Yao, J. Design, simulation and testing of a novel radial multi-pole multi-layer magnetorheological brake. Smart Mater. Struct. 2018, 27, 025016. [CrossRef]

13. Zhang, P.; Dong, Y.; Choi, H.J.; Lee, C.-H. Tribological and rheological tests of core-shell typed carbonyl iron/polystyrene particle-based magnetorheological fluid. J. Ind. Eng. Chem. 2018, 68, 342-349. [CrossRef]

14. International Standards of Medical Electrical Equipment, IEC 60601-1, 3rd ed.; International Electrotechnical Commission: Geneva, Switzerland, 2005.

15. Electromagnetic Brakes. Available online: https://www.mikipulley.co.jp/EN/Products/ ElectromagneticClutchesAndBrakes/ (accessed on 22 September 2019).

16. Mekid, S. Spherical Joint with Internal Brake. U.S. Patent 8,608,398, 17 December 2013.

17. Iqbal, H.; Yi, B.-J. A New Design of Spherical Electro-Magnetic Brake System. In Proceedings of the 2018 15th International Conference on Ubiquitous Robots (UR), Honolulu, HI, USA, 26-30 June 2018; pp. $220-222$.

18. McLyman, C.W.T. Transformer and Inductor Design Handbook, 3rd ed.; Marcel Dekker Inc.: Hoboken, NJ, USA, 2004; pp. 262-263.

19. Schimpf, P.H. A Detailed Explanation of Solenoid Force. Int. J. Recent Trends Eng. Technol. 2013, 8, 8.

(C) 2019 by the authors. Licensee MDPI, Basel, Switzerland. This article is an open access article distributed under the terms and conditions of the Creative Commons Attribution (CC BY) license (http://creativecommons.org/licenses/by/4.0/). 